\title{
Zipper-Featured $\delta$-Peptide Foldamers Driven by Donor-Acceptor Interaction. Design, Synthesis, and Characterization
}

\author{
Xin Zhao, ${ }^{\dagger}$ Mu-Xin Jia, ${ }^{\ddagger}$ Xi-Kui Jiang, ${ }^{\dagger}$ Li-Zhu Wu, ${ }^{\perp}$ Zhan-Ting Li, ${ }^{* \dagger}$ Guang-Ju Chen ${ }^{*}$, \\ ${ }^{\dagger}$ Shanghai Institute of Organic Chemistry, Chinese Academy of Sciences, 354 Fenglin Lu, \\ Shanghai 200032, China \\ ${ }^{\ddagger}$ Department of Chemistry, Beijing Normal University, Beijing 100875, China \\ ${ }^{\perp}$ Technical Institute of Physics and Chemistry, Chinese Academy of Sciences, Beijing \\ 100101, China \\ E-mail: ztli@mail.sioc.ac.cn
}

\section{Supporting Information}

1) Experimental general (S2)

2) Partial NOESY spectrum $(400 \mathrm{mHz})$ of tripeptide $\mathbf{1 b}(30 \mathrm{mM})$ in $\mathrm{DMSO}_{-} \mathrm{d}_{6}(\mathrm{~S} 3)$

3) Partial NOESY spectrum $(400 \mathrm{MHz})$ of tripeptide 1c $(30 \mathrm{mM})$ in $\mathrm{DMSO}^{-\mathrm{d}_{6}}(\mathrm{~S} 4)$

4) Partial NOESY spectrum (400 MHz) of tetrapeptide $1 \mathbf{d}(10 \mathrm{mM})$ in $\mathrm{DMSO}_{-} \mathrm{d}_{6}(\mathrm{~S} 5)$ 
Experimental General. Melting points are uncorrected. Unless otherwise indicated, all starting materials were obtained from commercial suppliers and were used without further purification. All solvents were dried before use following standard procedures. All reactions were performed under an atmosphere of dry nitrogen. The ${ }^{1} \mathrm{H}$ NMR spectra were recorded on 600, 400, or $300 \mathrm{MHz}$ spectrometers in the indicated solvents. Chemical shifts are expressed in parts per million $(\delta)$ using residual solvent protons as internal standards. Chloroform $(\delta$ $7.26 \mathrm{ppm})$ was used as an internal standard for chloroform- $d$. 
Partial NOESY spectrum $(400 \mathrm{mHz})$ of tripeptide $\mathbf{1 b}(30 \mathrm{mM})$ in DMSO- $\mathrm{d}_{6}$ :

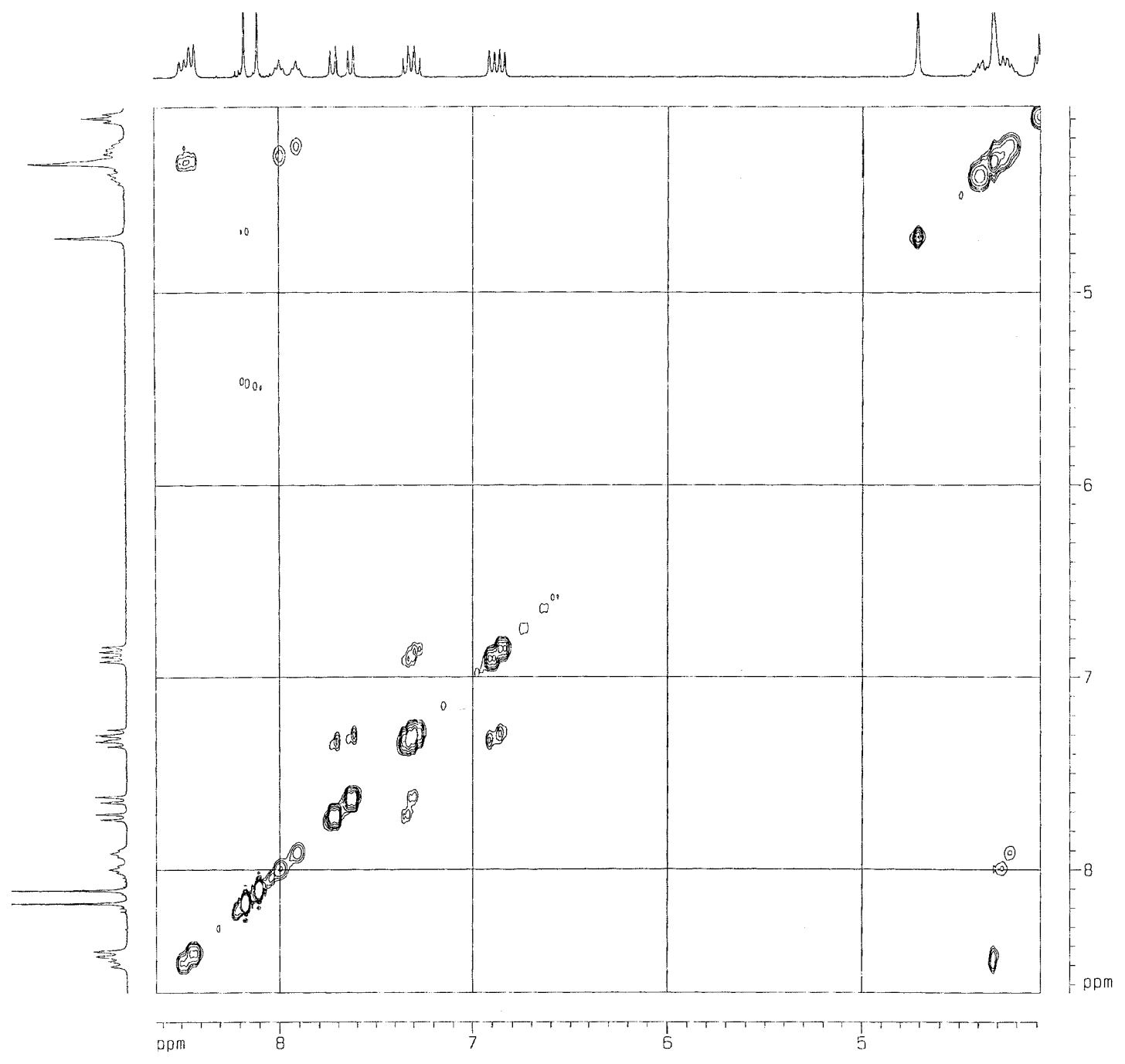


Partial NOESY spectrum $(400 \mathrm{MHz})$ of tripeptide $1 \mathrm{c}(30 \mathrm{mM})$ in DMSO-d $\mathrm{d}_{6}$ :

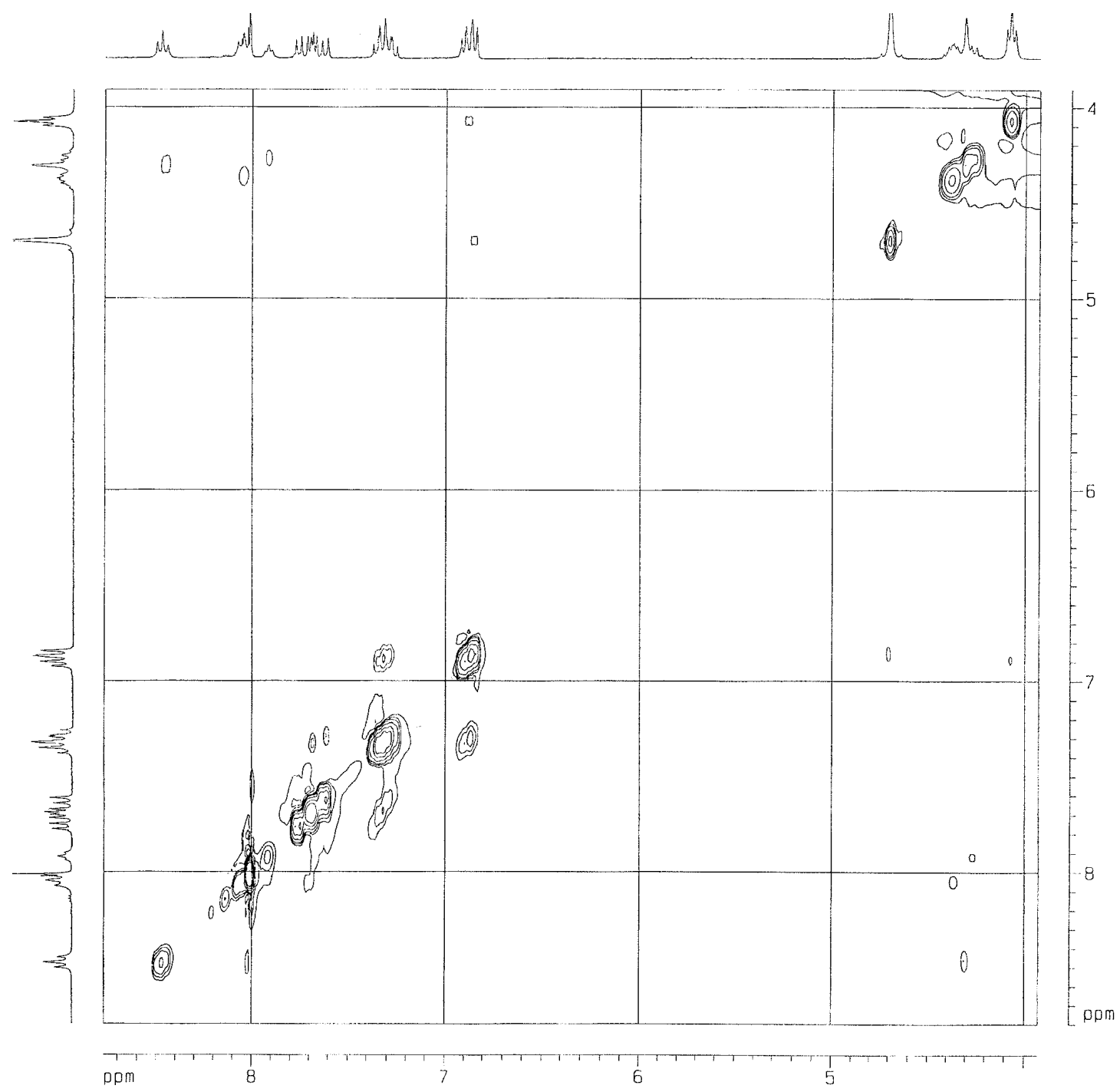


Partial NOESY spectrum $(400 \mathrm{MHz})$ of tetrapeptide $1 \mathbf{d}(10 \mathrm{mM})$ in DMSO-d $\mathrm{d}_{6}$ :

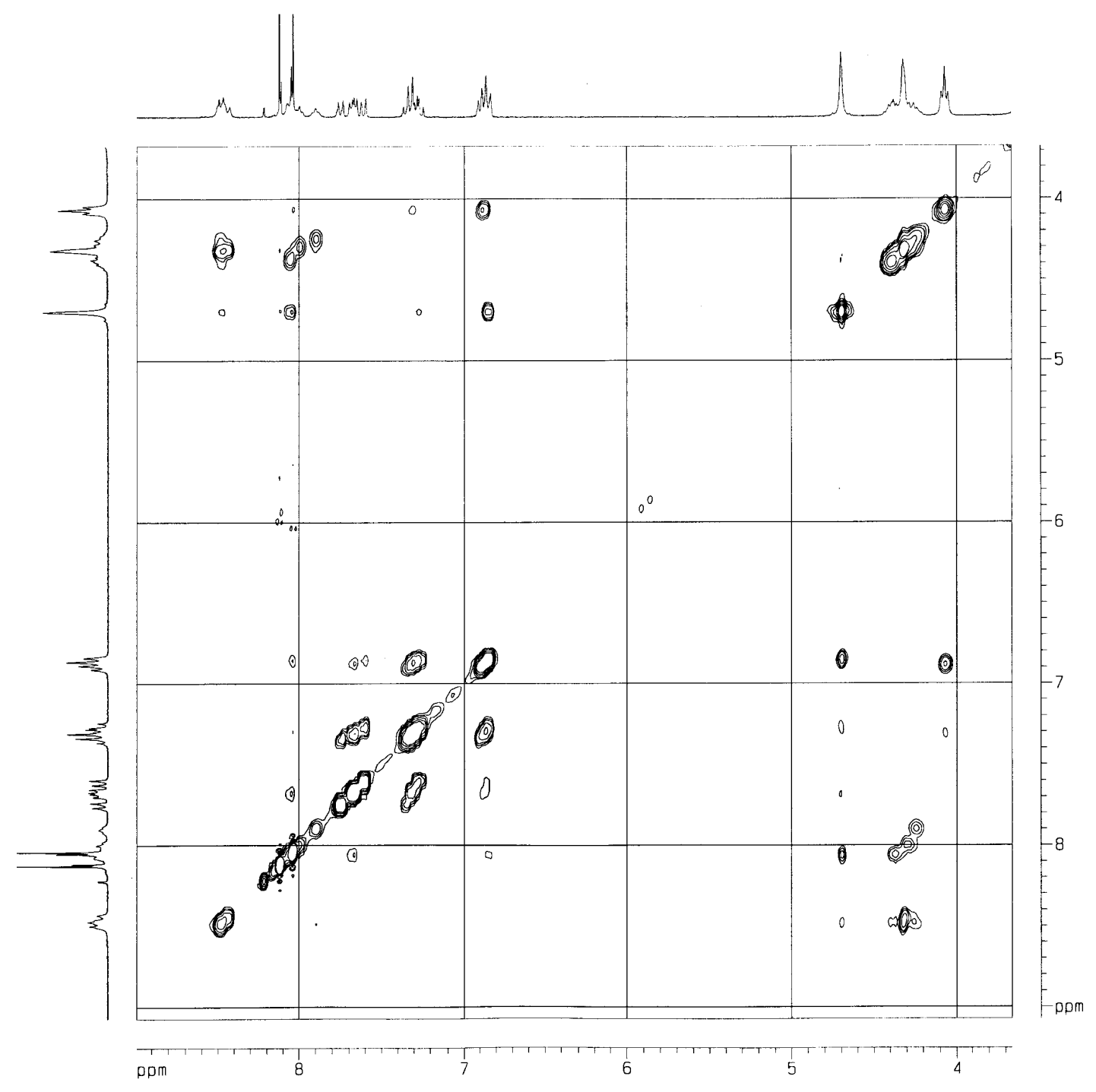

